\title{
Supporting Coordinated Care: Designing Social Context Visualizations for Care Teams*
}

\author{
T. Erickson, J. B. Ellis and K. P. McAuliffe \\ IBM T. J. Watson Research Center
}

\begin{abstract}
Coordinated care is a paradigm in which a multi-disciplinary team takes a holistic, proactive stance towards delivering care that encompasses both health and social issues. It focuses on understanding clients in their social contexts so as to anticipate problems and devise solutions that work within those contexts. For example, prescribing insulin to a diabetic will fail if the client lacks a refrigerator in which to store insulin, lives with someone who is likely to steal needles, or lacks the means to travel to the pharmacy. Social and health issues are intertwined. The effectiveness of coordinated care requires access to health and social data that enable care teams to understand clients' situations, assist them in interacting with their clients, and guide them in developing apt solutions. However, today's Electronic Health Records (EHRs) are ill-suited to these ends. This paper describes the setting, rationale, and design of social context visualizations for EHRs that combine health and social data. In particular, it describes two visualizations that show the client, the client's family and friends, and related information that enable a care team to quickly understand their client's social context.
\end{abstract}

\section{Introduction}

The standard approach to health and social care in much of the world has been problem-oriented. A mother becomes ill and goes to the doctor; an elderly person, disoriented and found wandering in the street, is aided by a mental health specialist; a child suffers abuse and is assisted by child welfare workers. Problems occur; care providers react. [1]

Over the last two decades, various health and social services agencies have been exploring a new paradigm for delivering care [2]. Though operating under various rubrics - coordinated care, patient-centered medical homes, community health teams, and integrated care - these approaches share a longer-term, more-proactive, holistic stance: care is delivered by multidisciplinary teams; problems are identified early when prevention is less costly; solutions are designed and managed to function in the context of a person's life. Coordinated care also treats those receiving care as active and empowered participants rather than passive recipients; as a consequence, and in line with the convention in social care, this paper refers to them as "clients" rather than "patients."

An important aspect of delivering care in this new paradigm is to enable a team to quickly understand a client's situation. The factors that characterize clients' situations - which will be referred to as their "social context" - are complex and intertwined. With whom does the client live? How does the client get along with others in their households? Does another household member have a problem that affects the client (e.g., illness, substance abuse, or special needs)? Are there environmental issues (e.g., mildew, pollution, or secondhand smoke) associated with the physical setting? And so on. In general, social factors can affect health directly, by

\footnotetext{
* To appear in IBM Journal of Research and Development, in press, 2014.
} 
contributing to health problems, or indirectly, by impeding solutions. The converse holds as well: health factors can have social impacts, such as a health problem leading to job loss or interfering with the ability to participate in a retraining program.

Another aspect of the coordinated care paradigm is that it makes use of both formal resources (i.e. those provided by government and institutions) and informal resources (e.g., support from family members, friends, and community groups and services). Thus, a care team member might recruit a friend to drive an elder to a medical checkup or identify a member of an extended family that could mentor an at-risk young person. A side effect of using informal resources is that it maintains ties with family, friends and community organizations that can better meet the individual's needs, and that replace costly professional services. [3]

The information needed to support the coordinated care paradigm, however, is not easily gleaned from Electronic Health Records (EHRs). In part this is because the information is produced and consumed by people in different institutions using different systems: health and social data are siloed, and cannot be readily integrated. And, in part, it is because EHRs are narrowly focused on clients themselves, and do not systematically represent knowledge about a client's household or family. [4] While the latter can only be addressed over time as systems and institutional practices and procedures co-evolve, systems are at a point where they can begin to support the integration of data from disparate systems.

The focus of this paper is on how to represent a client's social context as part of an EHR. Specifically, how can intertwined health and social data be presented and structured so as to assist the care provider teams in anticipating problems and designing solutions that work in the context of their clients' lives? This paper addresses this question by doing four things. First it characterizes the domain of coordinated care delivery. Second, it translates the domain understanding into a design rationale and embodies it in a rough prototype. Third, it evaluates the resulting prototype. Fourth, it describes an implemented version of the visualization customized to support the delivery of elder care in a district of Beijing. While the work described here represents the early stages of work in an ongoing project, its domain characterization, rationale, and design concepts lay out an important direction for development that needs more attention.

\section{Background}

\section{Prior Work}

Human-computer interaction (HCI) has devoted increasing attention to healthcare over the last two decades. In part, this has been driven by the adoption of EHRs, which has led to extensive research on the nature of the healthcare work, on the ways in which the design of EHRs facilitates or inhibits work, and on the design of new types of EHRs (e.g., [5, 6]). Another effect of the shift to EHRs is the creation of vast troves of data, and this in turn has encouraged investigations into data mining and population-level analyses (e.g., [7, 8, 9]).

Of primary relevance to this paper is work on visualization of healthcare data - [10] provides an overview of this work and a list of nine challenges for the future. Two strands of visualization research are particularly relevant. First, visualizations are being used in conjunction with narrative - both as a way of telling stories with data (e.g., [11]), and as means for eliciting 
narratives from clients (e.g., [12]). These resonate with the aims of this work - namely creating visualizations that enable care workers to quickly make sense of a client's situation, and that suggest conjectures that can be used to guide care worker-client interactions.

Second, while most work on visualizing health data has focused on the information for a single person, one line of work has explored ways of visualizing genealogical data - see [13] for an excellent review. In particular, [13] offers a number of cogent critiques of the use of traditional tree-like structures, including that they do not scale well and do not show temporal attributes or complex relationships well. While these critiques are well-taken, the work discussed in this paper nevertheless uses (in part) a tree-like visualization. The requirements of the coordinated care domain mitigate the critiques of tree-like structures for three reasons. First, the need for scaling is limited, as the material of interest is a few generations of a family. Second, the relationships emphasized in a family tree have social meanings (e.g., norms, roles, expectations) attached to them. Third, the family tree structure is familiar to a wide range of people. These last two points relate back to the aim of supporting narrative and sense-making.

With respect to the coordinated care paradigm, there is less research in HCI. Most of what is relevant takes as its starting point the notion that patients take an active role in their own care, and that prevention and the maintenance of good health is the aim. This has led to two lines of work. One has to do with providing individuals with tools that allow them to monitor their health and engage in self-directed behavior change to maintain wellness; this has been fueled by increasing availability and use of personal and embedded sensors and mobile devices (e.g., [14]). A second, related line of work has looked at ways in which family, friends and other informal support resources can support individuals in these endeavors, through group activities, wellness games and online communities (e.g., [15, 16]). A particularly apt project is CareNet [17], which focuses on providing information to the informal support networks of family members and friends involved in elder care. To the best of our knowledge, there is no work on designing visualizations to support the formal side of delivering coordinated care: namely, the multidisciplinary teams charged with care delivery.

\section{This Project}

This work is part of a larger project that explores the integration of data from various health and social care data sources. This is challenging because the data is heterogeneous and distributed across different systems; furthermore, the data in question is often owned by different institutions with different requirements for access control. The technical response to this challenge is to use semantic technologies that use explicit semantics and explicit models for data representation and search; the solution uses a three layer architecture a virtual RDF view layer, a distributed query processing layer, and a unified context view layer. More details can be found in $[18,19]$.

The work discussed in this paper assumes that the technical challenges of data access have been addressed, and focuses on how to visualize large quantities of diverse data. It is important to carry out this work in parallel with the more technical work, because the requirements of presenting data has implications for what is gathered, and how it is integrated and managed.

A second aspect of the work described in this paper is that it is not pure research, but rather is carried out as part of a client-driven project. The chief consequence of this is that some of the 
methodological choices made in carrying out this research are dictated by practical constraints like client needs, time tables, and logistics. For instance, the last stage of work, the deployment of an implemented prototype in Beijing, was not ideal for research purposes - it introduced new challenges due linguistic, cultural and institutional differences - but nevertheless, that is where the larger project was able to find a client interested in deploying the technology.

\section{Methods}

The methods used in this paper are from the field of human computer interaction, and are often referred to as user centered design or interaction design (e.g., [20]). In brief, work proceeds by gathering information about the domain (e.g., characteristics, tasks, user needs), representing the interactions to be supported in design artifacts (e.g., user scenarios, use cases, rough prototypes), and seeking feedback on both the gathered information and the design artifacts. In this approach - sometimes referred to as "design research" or "research through design" - results are embodied as design artifacts that are useful to both researcher and practitioners, rather than as hypotheses that are accepted or rejected. [21]

This process does not follow a waterfall model where one stage is completed before moving on to the next, but rather a spiral model where early cycles of the information gathering-designfeedback cycle are executed quickly and roughly, and subsequent cycles take place more slowly as the domain becomes better understood, the design artifacts more detailed, and the feedback sought more precise. The work in this paper proceeds through several turns of the cycle, and falls into two general phases, the first being initial design work, and the second being the development of an implemented version for an on-the-ground deployment in Beijing.

\section{The Coordinated Care Domain}

The initial design work began with visits to two practices operating in the coordinated care paradigm. Members of the design team met with both managers and care workers; the design team also interviewed other subject matter experts (SMEs) with experience in both medical and social care delivery, and made use of other resources they suggested.

A number of themes recurred in both the interviews and the literature. One was that being reactive is expensive. A common story featured a person with a chronic disease like asthma or diabetes or who is in and out of a hospital; while the protagonist's immediate problems are treated, the root problem is not solved and the problem recurs. The moral offered for this story is that in the long run it may be much more cost effective to address the root problem than to pay for repeated hospitalizations.

A second, related theme was that often the specifics of a client's situation disrupt the accepted ways of addressing a condition: "It's hard to treat diabetes if you can't take care of your insulin, if you can't get your syringes, if you can't get to the pharmacy, if you don't have transportation, or if you can't keep track of those things. [01:18]" [22]. Problems like these may not be evident; furthermore, many clients are embarrassed and reluctant to volunteer such information - it may only emerge gradually as trust is established through extended conversation. One Community Health Team noted that its important to have a detailed understanding of their clients' situations 
to be able to develop solutions that work. For example, it may not enough to enroll a client in a program; it may be necessary to ensure that they have transportation to get there.

A third theme was that many problems are systemic. Allergies may be due to mildew in substandard housing - moving rather than pills may be the solution. Loss of employment may lead to substance abuse and thence to domestic violence - employment counseling may be more effective than anger management classes. Overall, it's vital to understand the systems in which a problem is embedded to be able to design appropriate solutions.

A fourth theme was that multi-disciplinary teams (MDTs) and collaboration are central to coordinated care. First, members of MDTs are diverse - from care navigators who understand government programs and eligibility, to social workers, to medical personnel. Second, decisionmaking is team-based: 'Collaboration is an intrinsic part of everything,' as one of the SMEs said. Third, a consequence of having a team supporting a client is that each team has many clients to keep track of. Fourth, the collaborative approach includes the clients: they, and often their family, need to 'buy into' an approach. Sometimes, as one informant said, this may mean that the first problem to be tackled isn't the most urgent one, but the one that the client feels able to address. Small successes early are important. This means that on-going conversation between a client and members of his or her care team is crucial.

\section{The Design Rationale and Rough Prototypes}

The results of the domain investigation were translated into a design rationale for guiding the design of the visualizations. The rationale had three principle elements:

- Broadly accessible. MDT members are diverse. The visualization must not rely on disciplinespecific knowledge.

- Glanceable. MDTs have many clients: they need to rapidly make sense of a new client's situation, and be quickly reminded of clients they've worked with before.

- Systemic analysis support. The visualization should help its users understand the systemic nature of health and social problems so that solutions devised by the MDT are compatible with the clients' situations.

The development of the social context visualization began with a type of hand-drawn diagram called a genogram [23]. Genograms emerged from an area of social work called family therapy in the mid-1980s $[23,24]$, and were adopted in various areas of social and health work over the next several decades [25].

Genograms resemble family trees with complex symbolic annotations, and provide information about family members' relationships and problems. Thus, the genogram shown in Figure 1 shows a divorced couple, Carl and Cindi, with 2 children who live with their mother (indicated by the dashed boundary), the couple's siblings and parents, and the ages of all concerned. It also shows atypical emotional relations among family members (shown by jagged and parallel lines), and challenges like mental illness and substance abuse (shown via half shading of entities) and obesity, diabetes and smoking (indicated via alphabetic notations like O, D and S). 


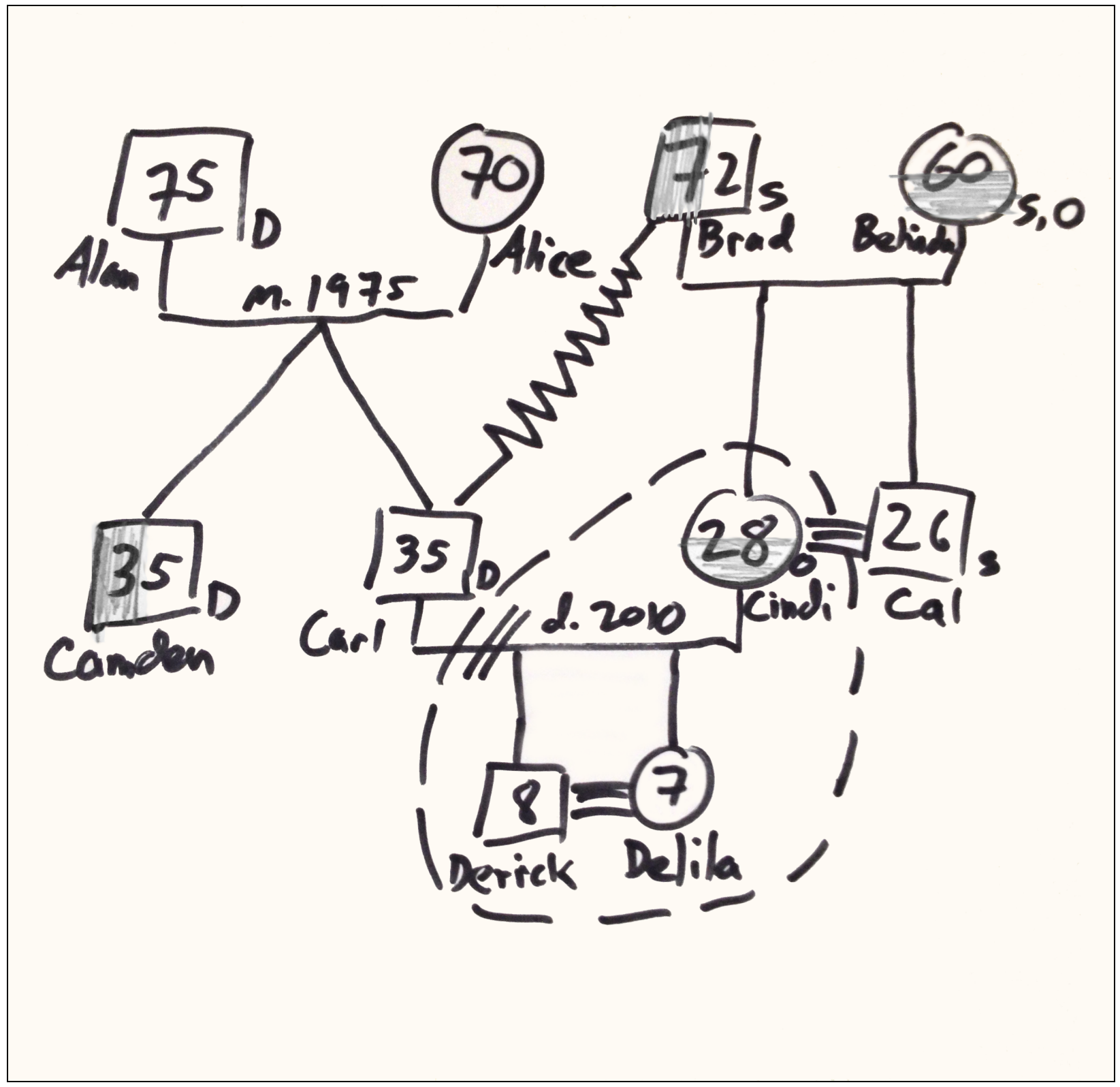

Figure 1. A genogram of Cindi, showing her household (circumscribed by the dashed line), and her extended family her ex-husband, two children, and her and her ex's parents. The shapes (square for male, circular for female) indicate family members with their names and ages, the single straight lines indicate kinship or marital relationships (a double slash depicts the divorce), and the more complex lines indicate abnormal emotional relationships. Halfshaded shapes indicate mental or physical illness issues, and single-letter annotations indicate problems like diabetes, obesity and smoking.

Genograms have pros and cons $[23,24]$. They were a good place to begin because they captured many types of data in a family-centric representation. As a representation that originated in the field of family practice, and that evolved to support a range of uses in different social and healthcare fields, it seemed likely that they would support a broad range of informational and analytical tasks. In particular, in keeping with the goal of supporting systemic analysis, they had been developed by a discipline concerned with problems rooted in the dynamics of families, and that viewed problems as the outcomes of systems of behavior. 
At the same time, genograms were not an ideal fit. They evolved as hand-drawn diagrams, not as interactive representations, and had developed an arcane symbology. They had grown to include 15 relation types to designate emotional relationships such as "caretaker" and "fused," 7 node types indicating gender-orientations, 7 node patterns indicating various mental and physical issues, and a range of other annotations that were useful for family analysts, but less accessible to others. Adaptation was needed.

The rationale guided the development of a series of rough prototypes of social context visualizations (SCV). To make the SCV broadly accessible, the genogram was simplified by adopting a conventional family tree structure, reducing emotional relation types from 15 to simply positive or negative, replacing node types and patterns with pictures/icons for people, and providing annotation layers that could be turned on and off. Tooltips were used to reveal more detailed information about nodes and links between nodes, details about marital relationships, and to describe the challenge and strength icons associated with people.

The use of the more conventional family tree diagram and the simplification of the genogram's visual conventions also served to make the SCV glanceable, as did the use of photos and toggleable layers. The intent was that a care worker be able to quickly view who was in the household, expand it to the extended family, and get a sense of the magnitude of problems.

Systemic analysis was supported by the use of the family tree structure, and challenge/strength icons attached to tree nodes. Family therapists have a sophisticated understanding of family dynamics - e.g., that dysfunctional emotional relations can repeat across generations - and this representation makes such multi-generational patterns visible. Similarly, making the associations among groups of people visible - whether it be cohabitation, familial relations, or emotional connections, enables the diagnosis of environmental, emotional or social problems. It also provides a basis for making inferences about possible sources of support within the household or extended family. All of these reflect ways in which the original hand-drawn genogram was used.

\section{Rough Prototype Evaluation}

The design rationale was embodied in a series of rough prototypes, each of which was critiqued - initially by the design team, and later by SMEs - and used to refine the design. After the design ideas had stabilized, they were embodied in a simple interactive mockup that was used to evaluate the design with surrogate users.

The interactive mockup (Figure 2) enabled users to use the SCV to explore information about the social context of two clients. The mockup enabled its users to view the client's household or extended family (upper and lower views, respectively) and hide or reveal emotional relations, and challenges and strength (by using the controls along the top). Positive emotional relationships were shown as solid lines and negative relations as dashed lines, and challenges and strengths as square icons associated with each individual. Tooltips were used to reveal information about the various entities in the visualization when users hovered over them (several examples of tooltips are shown in the lower view, although in actual usage only one tooltip would appear at a time). 

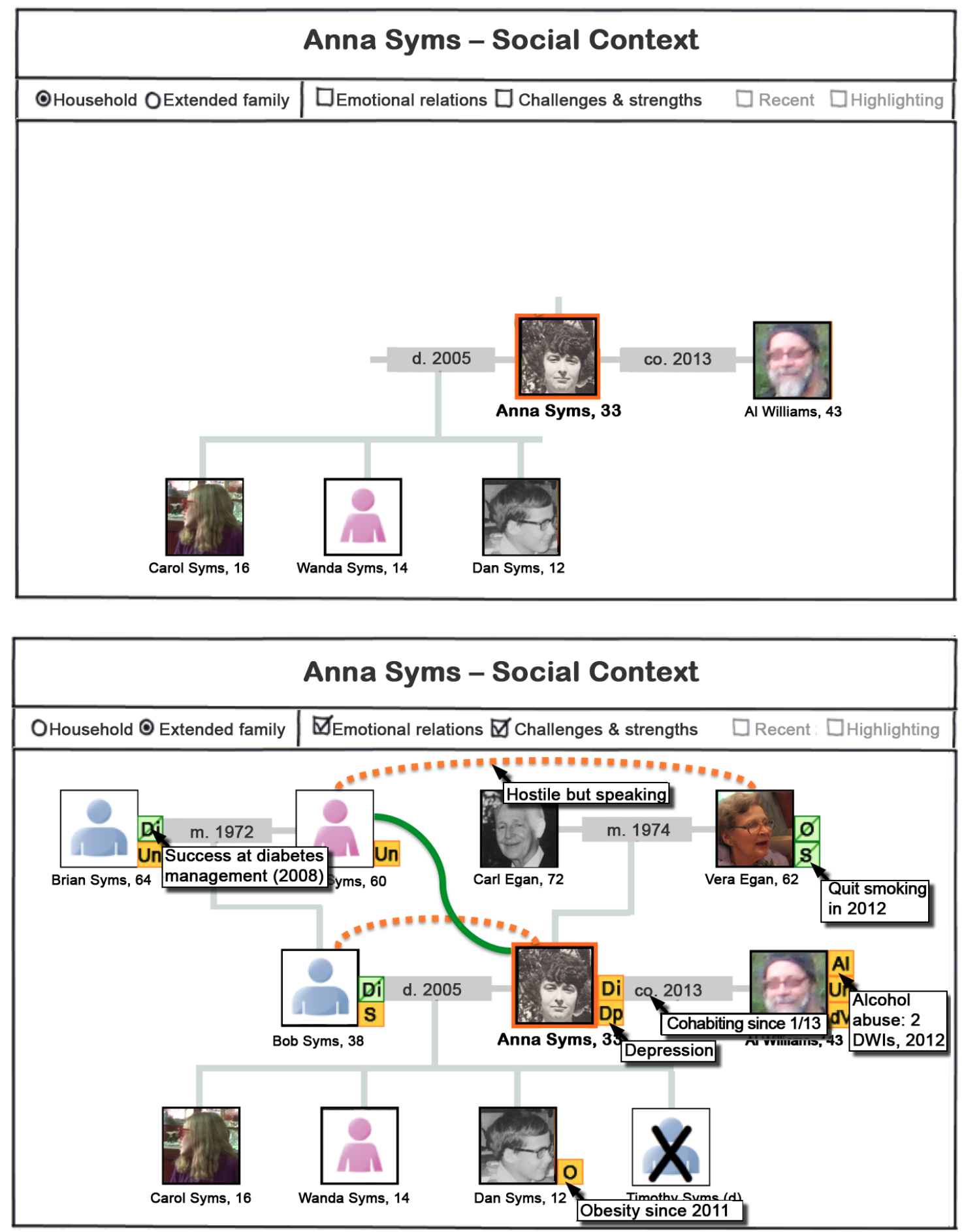

Figure 2. Two views of Anna Syms' social context from the interactive visualization mockup. The upper view contains only the household, showing names, photos and relationships among members of the household. The lower view shows the entire extended family, and adds layers that show positive and negative emotional relations (solid and dashed lines) and challenges and strengths (as square icons). Hovering reveals a tooltip with the details of relationships and other annotations (in actual use only one tooltip is shown at a time) 
The evaluation had three goals. The first goal was to determine whether users could figure out how to use the visualization without instruction. The second was to detect bugs in the user interface design. The third was to get a sense of the degree to which users could 'make sense' of clients and their situations.

The evaluation had two parts. In the first, evaluators were shown an SCV (for a different client than shown in Figure 2), informed that they were seeing a new type of client record, and asked to discover how to use it and to describe client 1's situation. They were not provided instructions on how to proceed, although if several minutes had passed without success (this did not occur) they would have been given hints of increasing specificity. In the second part of the evaluation, users saw a SCV for a second client, and were asked to role-play a care worker preparing to meet with the client in a few minutes. There were asked to describe what they understood about the client, and suggest some topics that should be discussed in the upcoming meeting. In both parts, evaluators were asked to think out loud; at the end of each case, there was a period of openended discussion.

The 8 evaluators, 3 women and 5 men, were recruited from among colleagues; 2 had exposure to social work, one as a longtime volunteer care worker, and the other via coursework. Colleagues were recruited both because it was convenient and because the SCV is intended to be used by a wide range of people, some of whom will lack training in medical and social work. Sessions lasted about 45 minutes; audio and screen recordings were captured with permission. One person ran the session; a second took notes.

The evaluation achieved its goals. First, evaluators had no difficulty understanding the visualization or learning how to manipulate it - no one required instructions. Second, while evaluators turned up various bugs in the design (e.g., no indication of the directionality of emotional relations), none of the bugs were sufficiently serious to hinder the use of the visualization, and all had simple remedies. Third, evaluators were able to use the SCV to get a sense of the client and her family. For instance, one commented that "the boxes [the orange boxes that indicate challenges] give me a good indication of where the heavy [emotionallycharged] stuff is" and "the more green boxes [strengths] I see, the more resilient the family is." He also speculated that the emotional relations spanning the two sides of the family indicated that it had been close before the divorce disrupted it.

It was clear that evaluators were adept at bringing their knowledge of families and interpersonal dynamics to bear when interpreting the visualization. For instance, in the case of Anna Syms (the client whose information is shown in Figure 2), one evaluator suggested that her depression might be due to the upcoming tenth anniversary of her youngest son's death, and another that it was her divorce. Many were concerned about Anna's relationship with Al, her cohabiter: Would his presence pose a financial burden? Would his alcohol abuse make him a danger to her children? And so on. All of these comments go beyond the information provided in the SCV they illustrate that the visualization enables evaluators to generate a narrative from the data. This is important, because it is this capability that the allows care team members to form hypotheses and to determine how to approach conversations with their clients. 
Another observation had to do with the absence of information. Evaluators often based their reasoning on the absence of information. Anna's depression may be situational (i.e. owing to the loss of a child), since neither parent was flagged as having had depression. At the same time, most realized that negative information could either mean "no" or "don't know." Making this distinction explicit - perhaps flagging "don't know" instances - could be useful, both in making sense of the situation and in soliciting information to 'fill in the blanks.' More troublesome to many was the absence of emotional relations - e.g., between Anna and her children. This is due to a convention, inherited from genograms, of only labeling unusual emotional relations; strong bonds between parents and children are assumed, unless otherwise marked. None of the evaluators (including those with a social work background) recognized this. Yet, addressing this by labeling all emotional relations adds a lot of complexity to the SCV; this is an area that requires more investigation.

A final observation is that, in trying to make sense of a situation, evaluators were often concerned with when things started, how long they persisted, and what had happened most recently. The SCV mostly presented information with dates, which evaluators would mentally subtract to determine durations; it would be straightforward to calculate this for them. " $A$ good social worker thinks in time," said one evaluator with experience in social work. When we asked if a timeline might be a more suitable form of visualization, he commented that he would rather stay with the family tree diagram. Timelines are fine for post mortems, he said, but the family tree diagram shows you where the resources are - what might be drawn upon to address a client's problem. He also strongly argued for including non-family members in the diagram, because it expands the range of social resources that are available, a direction taken up in the next phase of work.

\section{Designing a Working Prototype for Deployment}

Up to this point in project, the work had been quite general: it had focused on designing a way of representing information for multidisciplinary care teams who might be located anywhere, and might be caring for any type of client population. As described, it did this by drawing on expert informants, general accounts of the coordinated care domain and its needs, and interviews with various subject matter experts (SMEs).

To make further progress, it was necessary to implement a working version of the prototype. This in turn required a customer in the social and health care delivery domain that both had a need for such a system and was willing to engage in the time-consuming iterative work needed to customize, implement and deploy a working system on the ground.

The customer in question is involved in the delivery of social and health services for the elderly, and is located in a district of Beijing. It drew on four types of resources: personnel and services from the Home-based Care Department of a local hospital; personnel and services for the elderly from a local Community Services Center; for-pay services (e.g., meal delivery) provided by a commercial contractor; and support from family members and volunteers from the local community. 


\section{Method}

A key challenge for this part of the project is that the design team and customer were from different cultures, and thus had to work across linguistic and cultural gaps. To succeed, the team used written and graphical artifacts that used examples to make things concrete. Artifacts were created, shared, discussed and annotated - usually many times. The concrete nature of the artifacts aided the understanding of what was being proposed: the customer was able to identify things that were confusing or simply wrong, and the design team was able to develop an understanding of the work practices and settings in which the system would be used. The use of artifacts was important because collaboration usually occurred across a great distance, and linguistic limitations meant that real time communication was not always fluid. Having documents that could be read asynchronously and annotated enabled much finer-grained feedback.

The artifacts were of three types:

- User scenarios described the system from the point of view of the people using it, telling a story about a particular person that showed who was using the system, why they were using the system, what they were using it to accomplish, and where the system was being used.

- Use Cases specified the ways in which the system was used to accomplish particular tasks, and illustrated the steps a user would take to accomplish the task using the system.

- Mockups showed approximately what the user interface would look like, and were created to make sure that the use cases could be carried out by the users.

The process began with the creation of scenarios that illustrated the functionality to be supported. Three scenarios depicted elders in varying living situations (e.g., a widower with no local family; a widow living with her son and his wife), with different health and social challenges (e.g., a recently widowed and depressed elder; a woman with diabetes and difficulty walking). The scenarios were iterated on over about two months; initially they drew on generic research on the needs and requirements for elder care in China (e.g., [26, 27]) and on client documents (e.g., assessment forms; menus of services available to the elderly); as the scenarios became more detailed and concrete, the customer was able to provide feedback and additional input.

After the design team and customer agreed that the scenarios represented what was to be done, detailed use cases were developed. These were described textually, and then illustrated using sketches and mockups derived from the rough prototypes described previously. As with the scenarios, these were shared with clients, and iterative feedback was used to refine their features.

\section{The Working Prototype}

The working prototype was developed using HTML, CSS, and Javascript. The visualizations were built using the D3 javascript library. All the views are built client-side using data loaded dynamically from a REST API.

The working prototype includes two views: a family view, and a community view. The family view (Figure 3) is the descendent of the work discussed earlier in the paper, customized to support the needs of the eldercare situation. It differs from the earlier prototypes in three ways. First, it omits the ability to view a layer that shows the emotional relationships among people this is because the social and medical records being accessed to create the visualization do not 
have this information. Second, in response to suggestions from the customer, there are "group nodes" that show collections of people like siblings and children, the aim being to keep the representation compact by aggregating less central individuals. Third, rather than the "strength" and "challenges" icons shown in the previous prototypes, the working prototype shows icons denoting risk - significant problems the client is currently facing - and vulnerabilities - warnings of problems that the client may face later on. The risks and vulnerabilities are determined using predictive analytics using approaches described in [28].

Figure 3 shows an example of the family tree view for "Xianghua Wong," the 81-year-old widow who was the protagonist of one of the scenarios. Using the control at the upper left, users can flip between the household and extended family view, and quickly see that Xianghua lives alone. The halo of icons around Xianghua gives an immediate indication that she has a number of problems, real or potential. The rectangular icon above her photo contains the Chinese characters for "risk," and hovering over the icon would reveal that she is at risk for "requiring assistance for eating or getting out of bed." The smaller icons to the left of her photo indicate that she has a number of vulnerabilities, such as likelihood of losing sensory capabilities, difficulty performing daily activities, decreased social interaction, and depression. Exploring other nodes in the family tree, one can see that her husband is deceased (indicated by the grayed-out icon and superimposed candle), and that her husband has three siblings - with hovering revealing more details. It is also evident that Xianghua has three children, two of whom have children of their own. As with the earlier prototypes, the aim is to provide at-a-glance information about Xianghua, her living situation, and her extended family.

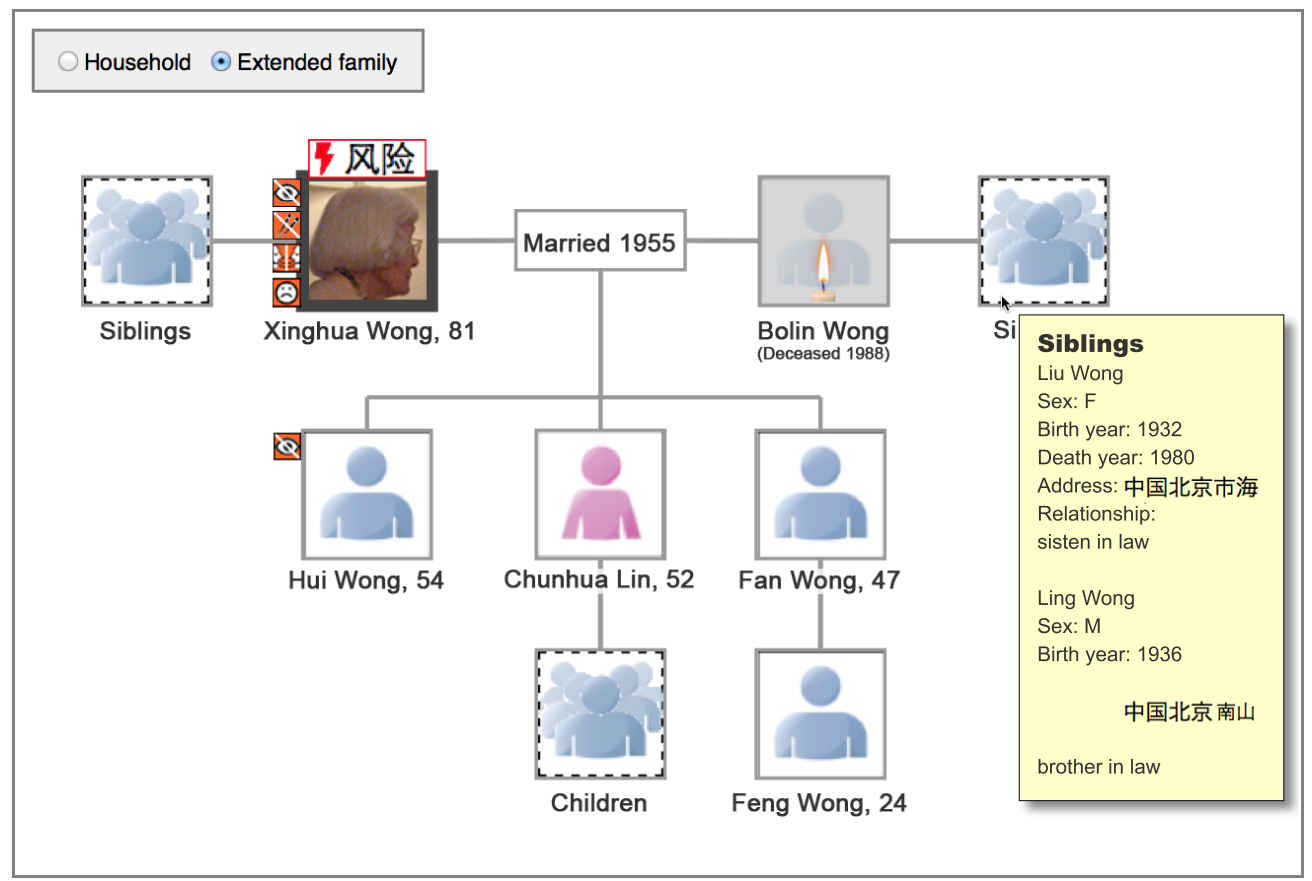

Figure 3. The implemented social context visualization: family tree view. This instance shows Xianghua Wong's extended family, and the risks and vulnerabilities family members have - i.e., Xianghua Wong has a risk (retangular icon), and she and her son Hui Chang have one or more vulnerabilities (smaller square icons). To keep the visualization compact, some members of the family (e.g., siblings; grandchildren) are represented by collective nodes (shown with a dashed border and a generic group icon). Hovering reveals details of family members and relationships. This view assists the care team in understanding the composition of the household and extended family, and the problems with which family members may be dealing. 
The second part of the visualization is the Community View. This is a response to the fact that eldercare in China depends on both volunteers and government workers: family and friends, volunteers, and services available for free or for purchase through the Community Services Center. It also responds to feedback from the rough prototype evaluation, emphasizing the importance of friends and others in the community as a source of informal support. The primary aim of the Community View is to show which resources are nearby so that they might be easily drawn upon.

Figure 4 shows the Community View with family, friends and volunteers, and services. It dispenses with family structure, and instead simply shows approximate distance of each person or service from the client. Because people may sometimes be far away, the visualization differentiates between those who are "nearby" - within a radius of 10 kilometers (or any value that the system administrator chooses to set) - and "far away." Those who are far away are shown outside the near/far radius (indicated by a dashed circle) and no attempt is made to show their proportional distance; within the radius, distance from the client is proportional to actual distance. This makes it easy to see whether there are nearby family members and friends who can be called upon for assistance. As with the other visualization, hovering over a visual element reveals detailed information.

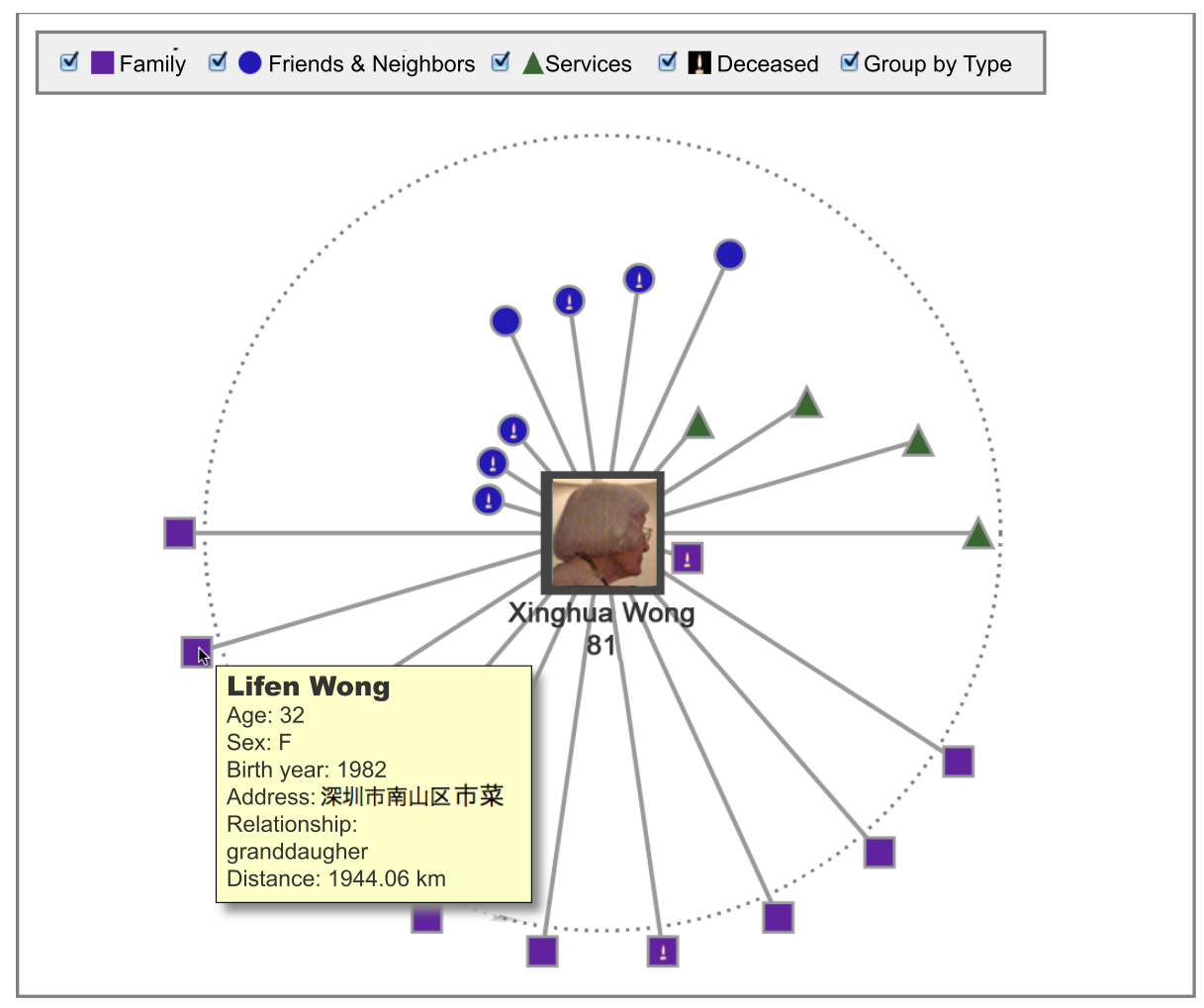

Figure 4. The implemented social context visualization: community view. This instance shows Xianghua Wong's family members (squares), friends and neighbors (circles), and services (triangles) according to their proportional distance; icons outside the dotted circle represent those who are "far away." As before, hovering reveals details. This view assists the care team in understanding who is 'nearby' and thus can potentially provide companionship or other forms of support. 
Thus, in Figure 4, one can see that although Xianghua Wong has a number of family members (the square icons), all of whom are far away (outside the dotted circle), except one who is deceased (as indicated by the candle flame icon). Similarly, the visualization shows that she has a few friends, only two of whom are alive, and makes use of four services. A care worker viewing this visualization might be prompted to ask questions - such as how frequently Xianghua talks with her remote family members - and to consider ways of increasing Xianghua's local network of friends. Actions to increase her network of friends could also help address her vulnerabilities involving depression and decreased social interaction.

\section{Next Steps}

The next stage of this work is to deploy the system in a township in Beijing. The visualization is currently implemented and translated into Chinese; work is proceeding to integrate it with a backend system that will provide access to the underlying data. Although the work with scenarios and use cases described here aims to ensure that the system will fit the work practices and address the needs of its users, decades of experience in human-computer interaction lead us to expect that unanticipated issues will arise, and they will in turn shape the evolution of the visualizations.

\section{Conclusion}

The design of systems to support the delivery of coordinated care is a complex problem. Health and social data needs to be integrated in a form that the diverse members of a multidisciplinary team can use. Ideally, care team members will be able to rapidly understand their clients' situations, and will be able to work with their clients to develop solutions that work within the constraints of their clients' situations. Unfortunately, as they exist today, Electronic Health Records offer little support for this type of work.

This paper has made three contributions towards supporting the delivery of coordinated care. First, it has characterized the coordinated care domain, outlining the nature of coordinated care and the needs of the teams that deliver it. Second, it has translated the domain understanding into design guidelines, and embodied them in a series of prototypes. Third, it describes two implemented visualizations that augment current EHRs in a proof of concept system.

There are several limitations to this work. First, while the early phases of design took general constraints into account, coordinated care is too broad an area for a single study to thoroughly cover. Second, the implemented visualizations are responses to a particular combination of care needs and institutional and cultural constraints; it will be important to deploy working prototypes in multiple contexts. Third, the implemented versions have not been yet been deployed, so issues involving interaction of the design with the constraints of daily practice remain to be explored. The bottom line is that generalization must be approached with caution, and that developing a general approach to supporting the delivery of coordinated care is a project that will necessarily span multiple research efforts in multiple domains.

While we see a wide range of issues that require further investigation, a particularly important line of future work has to do with supporting collaboration. Collaboration both within and among 
care teams is a central part of coordinated care. Coordinated care includes a variety of meeting types - intake meetings, differential response meetings, status review meetings, problem-solving meetings - each with particular requirements for support. Furthermore, care team collaboration may occur outside of organized settings as the team responds to emergencies - this will require support for teams separated by distance, and for non-team specialists who need to be brought in to deal with a crisis situation. Finally, as our capacity to gather different types of data grows - for instance, by mining public repositories of social network data - new types of data will need to be integrated. All of these demands place requirements on the visualizations, and on the user interfaces within which they are embedded.

\section{Acknowledgements}

Thanks to Ronan Rooney and Walter Sedlazek for introducing us to the problem that drove this work, and for feedback, suggestions, and great dialogue.

\section{References}

[1] IOM (Institute of Medicine). 2001. Crossing the Quality Chasm: A New Health System for the 21st Century. Washington, D.C.: National Academy Press. Available: http://www.iom.edu/ /media/Files/Report\%20Files/2001/Crossing-the-QualityChasm/Quality\%20Chasm\%202001\%20\%20report\%20brief.pdf

[2] World Health Organization. Integrated Health Services - What and Why? Technical Brief No. 1, 2008. [Online]. Available: http://www.who.int/healthsystems/service delivery techbrief1.pdf

[3] [A] Wiener, Joshua. The role of informal support in long-term care. In Key Policy Issues in Long-term Care. World Health Organization. January 2002. pp 3-24. Available: http://www.who.int/chp/knowledge/publications/policy_issues_ltc.pdf

[4] National Institutes of Health National Center for Research Resources. Electronic Health Records Overview. April 2006. http://www.himss.org/files/HIMSSorg/content/files/Code\%20180\%20MITRE\%20Key\%20 Components\%20of\%20an\%20EHR.pdf

[5] G. Fitzpatrick and G. Ellingsen, "A review of 25 years of CSCW research in healthcare: Contributions, challenges and future agendas," Computer Supported Cooperative Work, vol. 22., no. 4-6, pp. 609-665, 2013.

[6] C. Plaisant, B. Milash, A. Rose, S. Widoff, B. Shneiderman, "LifeLines: Visualizing personal histories," in Proc. CHI '96, New York, 1996, pp. 221-227.

[7] J. J. Caban, and D. Gotz, "2011 Workshop on visual analytics in healthcare: Understanding the physician perspective," in SIGHIT Record, New York, 2012, vol. 2, no. 1, pp 29-31.

[8] A. Rind, T. D. Wang, W. Aigner, and S. Miksch, "Interactive Information Visualization to Explore and Query Electronic Health Records," Foundations and Trends in HumanComputer Interaction, vol. 5, no. 3, 2013

[9] T. D. Wang, C. Plaisant, A. J. Quinn, R. Stanchak, B. Shneiderman, "Aligning temporal data by sentinel events: Discovering patterns in electronic health records," in Proc. CHI 'O8, New York, 2008, pp. 457-466. 
[10] B. Schneiderman, C. Plaisant, and B. W. Hesse, "Improving Healthcare with Interactive Visualization," Computer, vol. 46, no. 5, pp. 58-66, 2013.

[11] E. Segel, and J. Heer, "Narrative Visualization: Telling Stories with Data," in IEEE Trans. on Vis \& Comp. Graphics, vol. 16, no. 6, pp. 1139-1148, 2010.

[12] B. K. Smith, J. Frost, M. Albayrak, and R. Sudhakar. "Facilitating narrative medical discussions of type 1 diabetes with computer visualizations and photography." Patient Education and Counseling, vol. 64, no. 1-3, pp. 313-321, 2006.

[13] N. W. Kim, S. K. Card, and J. Heer, “Tracing genealogical data with timenets," in Proc. Int. Conf. on Adv. Visual Interfaces, New York, 2010, pp. 241-248.

[14] M. Tentori, G. R. Hayes, and M. Reddy, "Pervasive computing for hospital, chronic and preventive care," Foundations and Trends in Human-Computer Interaction, vol. 5, no. 1, pp. 1-95, 2012.

[15] S. A. Ballegaard, T. R. Hansen, and M. Kyng, "Healthcare in everyday life: designing healthcare services for daily life," in Proc. CHI '08. New York, 2008. pp. 1807-1816.

[16] J. Huh, and M. S. Ackerman "Collaborative help in chronic disease management," in Proc. CSCW'12, New York, pp. 853-862, 2012.

[17] S. Consolvo, P. Roessler, B. E. Shelton, A. LaMarca, B. Schilit, and S. Bly. "Technology for Care Networks of Elders," vol. 3, no. 2, pp 22-29, 2004.

[18] S. Koutoulas, V. Lopez, M. Stephenson, P. Tommasi, W. Shen, G. Hu, M. L. Sbodio, V. Bicer, A. Kementsietsidis, M. M. Rafique, J. Ellis, T. Erickson, K. Srinivas, K. Mcauliffe, G. T. Xie, P. M. Aonghusa, "Coordinating social care and healthcare using semantic web technologies," in CEUR Workshop Proceedings: International Semantic Web Conference Posters \& Demos, Sydney, 2013, vol. 1035, pp. 169-172.

[19] W. Shen, G. T. Xie, K. Srinivas, A. Kementsietsidis, J. Ellis, T. Erickson, and K. Mcauliffe, "Promoting integrated social and medical care through semantic integration and context visualization," in Linked Data and Knowledge Graph: $7^{\text {th }}$ Chinese Semantic Web Symposium and $2^{\text {nd }}$ Chinese Web Science Conference, Shanghai, 2013, pp. 90-97.

[20] Y. Rogers, H. Sharp, J. Preece, Interaction design: Beyond human-computer interaction. Third edition. Chickchester, UK: Wiley, 2011.

[21] J. Zimmerman, J. Forlizzi, S. Evenson, "Research through design as a method for interaction design Research in HCI," in Proc. CHI '07, New York, pp. 493-502, 2007.

[22] P. Johnson, Hennepin County Health Care Reform video. [Online]. Available: http://www.hennepin.us/healthcare

[23] M. McGoldrick, R. Gerson and S. Petry, Genograms: Assessment and Intervention ( ${ }^{\text {rd }}$ Edition). New York: Norton, 2008.

[24] M. Scarf. Intimate Worlds: How Families Thrive and Why They Fail. New York: Random House, 1995.

[25] L. Paradopoulos, R. Bor, and P. Stanion, "Genograms in counselling practice: A review (Part 1)," Counselling Psychology Quarterly, vol. 10, no. 1, pp. 17-28, 1997.

[26] B. Wu, M. W. Carter, T. R. Goins, and C. R. Cheng, "Emerging Services for CommunityBased Long-Term Care (CBLTC) in Urban China: A Systematic Analysis of Shanghai s Community-based Agencies," Journal of Aging and Social Policy, vol. 17, no. 4, pp. 37-60, 2005.

[27] Y. Zhang, and F. W. Goza, "Who will care for the elderly in China? A review of the problems caused by China's one-child policy and their potential solutions," Journal of Aging Studies, vol. 20, no. 2, pp. 151-164, 2006. 
[28] J. Sun, J. Hu, D. Luo, M. Markatou, F. Wang, S. Edabollahi, S.E. Steinhubl, Z. Daar, and W.F. Stewart. "Combining Knowledge and Data Driven Insights for Identifying Risk Factors using Electronic Health Records," AMIA Annual Symposium Proc., 2010, pp. 901910.

\section{Biographical sketches}

Thomas Erickson IBM Research, Thomas J. Watson Research Center, P.O. Box 218, Yorktown Heights, New York 10598 (snowfall@us.ibm.com). Mr. Erickson is a social scientist and interaction designer in the Symbiotic Cognitive Systems laboratory at IBM Research. Originally trained as a cognitive psychologist, he joined a startup that developed software for the original IBM PC in 1982. After five years he moved to Apple, where he contributed to the design of the Macintosh user interface and worked on the development of various research prototypes, including Scribe, an ancestor of the iPad. In 1997 he joined IBM Research where he studies how people collaborate, both face to face and via technology, and applies this to designing new systems. He received IBM Outstanding Innovation Awards for his work in social computing in 2005 and 2011, has authored over twenty patents, published about eighty articles, edited two books, and is a Fellow of the ACM.

Jason B. Ellis IBM Research, Thomas J. Watson Research Center, P.O. Box 218, Yorktown Heights, New York 10598 (jasone@us.ibm.com). Dr. Ellis is a Research Staff Member in the Symbiotic Cognitive Systems Department at IBM Research. His work focuses on the design, implementation, and study of social technologies -- particularly those that facilitate collaboration among diverse user populations. Examples include social visualization for interdisciplinary caregiving teams, mobile collaboration in the developing world, online gaming communities, inter-generational communication, and distributed collaboration in open source development. He holds $10+$ patents and has published in top-tier HCI conferences such as Computer-Human Interaction, Computer-Supported Cooperative Work, Designing Interactive Systems, where he has also served on program committees. Dr. Ellis earned his $\mathrm{PhD}$ in Computer Science at Georgia Tech in 2003. Additional details available at http://jellis.org

Kevin P. McAuliffe IBM Research, Thomas J. Watson Research Center, P.O. Box 218, Yorktown Heights, New York 10598 (kpmac@us.ibm.com). Dr. McAuliffe joined IBM in 1984 at the Thomas J. Watson Research Center. Over his thirty years with IBM he has provided strategic, technical, and managerial leadership in IBM's Research, Sales and Distribution, and Software Divisions in a wide range of technical areas (including parallel processing, visualization, data integration, business process management and contextual computing) as well as for industry solutions (including healthcare, financial services, and social care). He works currently in Cognitive Knowledge Systems Department at the Thomas J. Watson Research Center. Dr. McAuliffe has received three IBM outstanding achievement awards for his contributions. He has co-authored over twenty patents and numerous publications. Dr. McAuliffe received his Ph.D. degree in Computer Science from New York University in 1986. 